\title{
Pozzi, protezione ambientale, salute pubblica
}

\author{
Nicola Cempini \\ Ingegnerie Toscane srl. \\ n.cempini@ingegnerietoscane.net
}

Il nostro Paese è caratterizzato dal fatto di avere gran parte del territorio molto antropizzato. L' approvvigionamento idropotabile è garantito in massima parte dalle acque sotterranee. Molto spesso aree caratterizzate da forti insediamenti civili e industriali si sovrappongono a un sottosuolo dove sono presenti gli acquiferi sfruttati.

Si tratta di una situazione molto delicata dove non è semplice trovare un punto di equilibrio tra le esigenze della civiltà e quella della protezione ambientale. In questo caso poi la protezione degli acquiferi dall'inquinamento non assume solo un valore ambientale in senso stretto ma si lega all'esigenza di salvaguardare la salute pubblica. Sono tragicamente note le conseguenze dell'inquinamento delle falde sulla salute delle persone.

Si tratta certamente, prima di tutto, di un problema culturale. Non solo gli operatori del settore ma i committenti dovrebbero essere coscienti che l'esecuzione di un pozzo rappresenta una perturbazione dell'ambiente naturale, sia che si tratti di un semplice pozzo a sterro, di un pozzo domestico di piccolo diametro, di un pozzo industriale o idropotabile di grande diametro. Per questo i pozzi devono essere progettati e realizzati seguendo rigorosamente la regola dell'arte: livelli acquiferi appartenenti alla stessa circolazione (acquifero multi falda) devono essere separati da adeguati tamponi e/o cementazioni; acquiferi relativi a circolazioni idriche diverse non solo devono essere separati da tamponi o cementazioni, ma devono essere messi in produzione con colonne diverse (pozzi multi colonna). Deve essere impedito qualsiasi contatto tra la circolazione idrica superficiale e quella profonda realizzando cementazioni e tamponi oppure (soluzione migliore nel caso di pozzi di profondità maggiori di 100-120 m) realizzando un avampozzo cementato a gravità o con sistema di iniezione.

Il corretto completamento del pozzo è importante poiché consente prima di tutto una migliore gestione dello stesso soprattutto in situazioni indesiderate: un livello acquifero che, magari dopo qualche anno di sfruttamento, denuncia un deterioramento della qualità dell'acqua, se correttamente tamponato, potrà essere escluso dallo sfruttamento con soluzioni tipo la posa di un packer o la cementazione interna. Se questa soluzione non è praticabile e si deve procedere comunque alla perforazione di un nuovo pozzo, l'esistenza di tamponi che separano i vari livelli consente all'idrogeologo di reperire informazioni fondamentali sulla qualità chimica, fisica ed eventualmente microbiologica di ogni livello permettendo una progettazione migliore. Se, ad esempio, siamo di fronte ad un pozzo che pesca da due livelli e quello superiore risulta deteriorato dal punto di vista qualitativo a causa di inquinamento antropico, si potrà procedere alla progettazione e realizzazione di un nuovo pozzo che escluda quel livello attraverso tamponi, cementazione o meglio ancora con l'esecuzione dell'avampozzo.

La separazione dei livelli produttivi, attuata con cementazioni e tamponi nei pozzi monocolonna o multi colonna, consente perciò interventi selettivi di manutenzione ordinaria o straordinaria. L'esecuzione dei tamponi e/o la posa in opera di più colonne consente inoltre all'idrogeologo di reperire maggiori informazioni e di maggior qualità sugli acquiferi in sfruttamento e sui loro rapporti.

Dal punto di vista legislativo la protezione delle risorse idriche idropotabili si fonda sulle cosiddette aree di salvaguardia.

Nel nostro Paese si parla per la prima volta di aree di salvaguardia dei pozzi a servizio del pubblico acquedotto con l'entrata in vigore del DPR 236/88 che attuava la direttiva CEE 80/778. Lart. 4 introduceva il concetto di aree di salvaguardia dividendole in area di tutela assoluta, rispetto e protezione.

Il decreto 236 non disponeva un iter preciso per la delimitazione di tali aree; ma le definiva in modo preciso:

1. area di tutela assoluta: 10 metri di raggio attorno all'opera che devono essere adibiti alle sole opere acquedottistiche, recintati e protetti;

2. area di rispetto: estensione minima di 200 metri di raggio attorno all'opera. Si trattava e si tratta ancora di un'area di dimensioni importanti: circa 12 ettari dove sono vietate tutta una serie di attività, quali gestione di rifiuti, apertura di altri pozzi (a meno che non siano destinati al medesimo approvvigionamento idrico), utilizzo di fertilizzanti, pascolo e stazzo di bestiame, dispersione da piazzali e strade di acque meteoriche e così via;

3. area di protezione: poche parole per quest'ultimo tipo di vincolo. Quest'area avrebbe dovuto essere destinataria di specifiche limitazioni in ordine a particolari attività.

L'individuazione delle aree di salvaguardia era demandata alle regioni (art. 9 comma f).

Larea di tutela di 200 metri di raggio poteva essere ridotta se se ne fossero ravvisate le condizioni "in relazione alla situazione locale di vulnerabilità e rischio della risorsa."

Il decreto legislativo 152/99 riprende sostanzialmente la definizione delle aree di salvaguardia già fornita dal decreto 236 sviluppando i vincoli inerenti l'area di rispetto e svilup- 
pando il concetto di zona di protezione che diventa un'area di salvaguardia dove bisogna disciplinare le attività antropiche in modo che non impattino sull'integrità quali-quantitativa della risorsa. Il decreto introduce soprattutto un iter preciso per la definizione delle aree di salvaguardia, ed in particolare nel caso della zona di rispetto la competenza viene demandata all'ambito Territoriale Ottimale, che deve proporre alla Regione la ridelimitazione dell'area di rispetto per l'istituzione della quale è perciò necessario un atto amministrativo regionale.

In mancanza della individuazione da parte della regione il decreto stabiliva che si dovesse mantenere in via provvisoria la delimitazione dei $200 \mathrm{~m}$ di raggio (criterio geometrico).

L'entrata in vigore del successivo testo unico ambientale, il dlgs 152/06, ha sostanzialmente confermato questa impostazione: l'area di rispetto deve intendersi individuata in modo provvisorio col criterio geometrico dei 200 metri di raggio. Alle ATO spetta di proporre alle regioni una diversa delimitazione che non significa per forza un restringimento.

L'accordo tra la Conferenza delle Regioni e lo Stato del 12 dicembre 2002 ha delineato la metodologia tecnica che deve seguire lo studio propedeutico alla definizione dell'area di rispetto individuando due criteri fondamentali:

- il criterio temporale;

- $\quad$ il criterio idrogeologico.

Il criterio temporale si fonda sull'individuazione delle isocrone (di solito a 60 e 180 giorni; area di rispetto ristretta e area di rispetto allargata) ed è quindi necessario modellizzare la falda per poter formulare una proposta in questo senso.. All'individuazione delle isocrone poi si deve abbinare la corrispondente realizzazione dei piezometri di guardia che consentiranno il monitoraggio. Il concetto è che se mai si individuerà un inquinante nel piezometro, il gestore avrà almeno 180 giorni di tempo per far scattare un piano di emergenza.

Il suddetto accordo suggerisce di utilizzare il criterio idrogeologico quando il contesto è troppo complesso per l'utilizzo di quello cronologico.
E' importante notare che un inquadramento idrogeologico di contesto è pur sempre essenziale per individuare correttamente l'area di rispetto. Ad esempio una falda in pressione, pur essendo separata dalla circolazione più superficiale da decine di metri di argille, può avere caratteristiche idrodinamiche tali che l'isocrona dei 180 giorni racchiude una porzione elevata di territorio che rischia di essere vincolato inutilmente.

Ho iniziato questo articolo parlando della necessità di eseguire tamponi e cementazioni a regola d'arte e ho proseguito ricordando brevemente quello che la legge prevede in termini di aree di rispetto dei pozzi.

La protezione delle risorse idriche parte infatti dalla corretta esecuzione dei pozzi; progettisti, direttori dei lavori e contractors devono prestare la massima attenzione al tema della salvaguardia ambientale. Un pozzo il cui completamento non abbia rispettato il sacro principio della separazione dei livelli acquiferi incontrati è un ottimo candidato a veicolare inquinanti tra falde diverse. Anche altri tipi di perforazione (pali, sonde geotermiche, dispersori, ecc.) devono essere eseguiti con criterio e assicurare il massimo grado di protezione ambientale.

Si pone quindi il problema delle aree di salvaguardia; occorre vincolare il territorio in maniera intelligente e non poliziesca. Le derivazioni idropotabili possono convivere nella maggior parte dei casi con un territorio fortemente antropizzato come il nostro. Occorre quando è possibile diversificare l'uso delle acque sotterranee, destinando al consumo umano le risorse maggiormente protette, limitando all'uso domestico (orto e giardini) le acque delle circolazioni più superficiali.

Si tratta senz'altro di corretta gestione del territorio e di pianificazione ma anche di selezione e formazione degli operatori. E' necessario che il mondo accademico e le associazioni di settore facciano proprio questo argomento e coinvolgano la politica in modo che un tema così importante per lo sviluppo e la salvaguardia dell'ambiente sia disciplinato in modo definitivo. 